\title{
MicroRNA-122 downregulates Rho-associated protein kinase 2 expression and inhibits the proliferation of prostate carcinoma cells
}

\author{
HONG LIU ${ }^{1 *}$, TENG HOU $^{2 *}$, WEN JU ${ }^{2}$, YIFEI XING ${ }^{2}$, XIAOPING ZHANG ${ }^{2}$ and JUN YANG ${ }^{2}$ \\ ${ }^{1}$ Department of Critical Care Medicine, Institute of Anesthesiology and Critical Care Medicine; ${ }^{2}$ Department of Urology, \\ Union Hospital, Tongji Medical College, Huazhong University of Science and Technology, Wuhan, Hubei 430022, P.R. China
}

Received May 27, 2018; Accepted January 17, 2019

DOI: $10.3892 / \mathrm{mmr} .2019 .9995$

\begin{abstract}
MicroRNA-122 (miR-122) has been reported to be involved in the pathogenesis of several types of malignancies; however, its role in prostate carcinoma remains unknown. Thus, the current study aimed to investigate the functionality of miR-122 in prostate carcinoma. Clinical data of 54 patients with prostate carcinoma who were diagnosed and treated in Union Hospital (Wuhan, China) between January 2011 and January 2013 were retrospectively analyzed. The expression levels of miR-122 and Rho-associated protein kinase 2 (ROCK2) in prostate tumor and adjacent healthy tissues of patients, as well as in the serum of prostate carcinoma patients and healthy controls, were detected by reverse transcription-quantitative polymerase chain reaction. Receiver operating characteristic curve and survival curve analyses were used to examine the diagnostic and prognostic values of serum miR-122 for prostate carcinoma. In addition, miR-122 mimic was transfected into prostate carcinoma cells, and the effects on cell proliferation and ROCK2 expression were explored by Cell Counting Kit- 8 and western blot assays, respectively. It was observed that miR-122 was downregulated and ROCK2 was upregulated in tumor tissues as compared with their levels in adjacent healthy tissues. miR-122 level in the serum was also markedly lower in prostate carcinoma patients in comparison with that in healthy controls. Furthermore, a low serum level of miR-122 was found to effectively distinguish the prostate carcinoma patients from healthy controls and to be an indicator of poor survival. In prostate carcinoma cells, miR-122 overexpression inhibited the
\end{abstract}

Correspondence to: Dr Jun Yang, Department of Urology, Union Hospital, Tongji Medical College, Huazhong University of Science and Technology, 1277 Jiefang Avenue, Wuhan, Hubei 430022, P.R. China

E-mail: slhwit1@163.com

*Contributed equally

Key words: prostate carcinoma, microRNA-122, Rho-associated protein kinase 2, proliferation, regulation proliferation and the expression of ROCK2. Taken together, miR-122 may inhibit the proliferation of prostate carcinoma cells possibly by downregulating ROCK 2 expression.

\section{Introduction}

Prostate carcinoma is a type of malignancy that develops from the prostate and one of the most common types of cancer in males (1). Prostate tumors usually grow slowly, without evident clinical symptoms at the early stages. Therefore, treatment of prostate carcinoma without distant metastasis usually yields satisfactory outcomes $(2,3)$. However, once metastasis affects other parts of the body, such as the lymph nodes, soft tissues, bone tissues or even the brain, mortality is comparable to that of other types of aggressive malignancies $(4,5)$. Mortality of metastatic prostate cancer ranks in the top among all malignancies in Europe (6). In developing countries, such as China, the incidence of prostate carcinoma exhibits an increasing trend, possibly due to lifestyle changes (7). Therefore, increasing the early diagnostic rate and improving treatment outcomes remains a major task for clinical personnel.

In spite of the lack of evident clinical symptoms during the early stages of the disease, the development of prostate carcinoma affects the expression of a large set of microRNAs (miRNAs or miRs) (8), indicating their involvement in the pathogenesis of this disease. miR-122 has been reported to serve a role as a tumor suppressor, inhibiting the progression of different types of cancer, including hepatocellular carcinoma (9) and oropharyngeal cancer (10). However, the involvement of miR-122 in prostate carcinoma remains unknown. Rho-associated protein kinase 2 (ROCK2) signaling serves pivotal roles in the development of various types of malignancies, including prostate carcinoma, and inhibition of ROCK2 inhibits the development of prostate carcinoma (11).

Our preliminary microarray data revealed the inverse correlation between expression levels of ROCK 2 and miR-122 in prostate carcinoma tissues (data not shown). Thus, in the present study, the aim was to examine the roles of miR-122 and ROCK 2 in this tumor. It was observed that miR-122 was downregulated in prostate carcinoma tissues, while miR-122 overexpression in prostate carcinoma cells led to inhibited proliferation and downregulated ROCK2 expression. 


\section{Materials and methods}

Specimens. Clinical data of 54 patients with prostate carcinoma who were diagnosed and treated at the Union Hospital, Tongji Medical College, Huazhong University of Science and Technology (Wuhan, China) between January 2011 and January 2013 were retrospectively analyzed. Cancer tissues, adjacent healthy tissues and serum samples of these patients were obtained. All tissues were stored in liquid nitrogen prior to use. The age range of these patients was between 27 and 71 years, with a mean age of $50.2 \pm 6.2$ years. Subjects were selected according to the following inclusion criteria: i) Patients with prostate carcinoma diagnosed through pathological examination; ii) patients with complete clinical and follow-up data; and iii) patients diagnosed and treated for the first time. The exclusion criteria were as follows: i) Patients who suffered from other malignancies at the time of the study or previously; ii) patients with other prostate diseases; iii) patients who had received treatment prior to admission; and iv) cases of mortality caused by other reasons. Furthermore, serum samples were also collected from 49 healthy volunteers who received routine physiological examinations in Union Hospital during the same time period to serve as the control group. The age of these heathy subjects ranged between 30 and 68 years, with a mean age of $49.2 \pm 6.3$ years. The age distribution of patients and healthy controls was similar. All participants and/or their families provided written informed consent prior to participation. The current study was approved by the Ethics Committee of Union Hospital, Tongji Medical College, Huazhong University of Science and Technology.

Cell culture and transfection. Human prostate carcinoma cell lines 22Rv1 (ATCC ${ }^{\circledR}$ CRL-2505 ${ }^{\mathrm{TM}}$ ) and DU145 (ATCC ${ }^{\circledR}$ HTB- $81^{\mathrm{TM}}$ ), and a human normal prostate epithelial cell line HPrEC (ATCC ${ }^{\circledR}$ PCS-440-010 ${ }^{\mathrm{TM}}$ ) were purchased from ATCC (Manassas, VA, USA). Cells were cultured with Eagle's minimum essential medium (ATCC) containing $10 \%$ fetal bovine serum (ATCC) in an incubator at $37^{\circ} \mathrm{C}$ with $5 \%$ $\mathrm{CO}_{2}$. A total of $5 \times 10^{5}$ cells in each well of a 6 -well plate were transfected with $10 \mathrm{nM}$ miR-122 mimics (HMI0068-5NMOL; Sigma-Aldrich; Merck KGaA, Darmstadt, Germany) or negative control mimics (HMC0002; Sigma-Aldrich; Merck KGaA) using Lipofectamine ${ }^{\circledR} 2000$ reagent (cat. no. 11668-019; Invitrogen; Thermo Fisher Scientific, Inc., Waltham, MA, USA). Overexpression of miR-122 in the transfected cells was confirmed by reverse transcription-quantitative polymerase chain reaction (RT-qPCR), and an overexpression rate between 190 and $250 \%$ was achieved prior to use in subsequent experiments. In case of ROCK 2 treatment, cells were treated with ROCK2 (5-554; cat. no. R1156; Sigma-Aldrich; Merck KGaA) at a dose of $10 \mathrm{ng} / \mathrm{ml}$ for $24 \mathrm{~h}$ after the transfection of miR-122 mimic.

Cell Counting Kit-8 (CCK-8) assay. The control and two tumor cell lines were collected during the logarithmic phase, and single cell suspensions with a cell density of $4 \times 10^{4}$ cells per $\mathrm{ml}$ were constructed. Next, $100 \mu \mathrm{l}$ of the cell suspension $\left(4 \times 10^{3}\right.$ cells) was added into each well of a 96 -well plate. Cells were then cultured at $37^{\circ} \mathrm{C}$ with $5 \% \mathrm{CO}_{2}$, and $10 \mu \mathrm{l} \mathrm{CCK}-8$ solution (Sigma-Aldrich; Merck KGaA) was added and mixed with cell suspension after 24, 48, 72 and $96 \mathrm{~h}$ of incubation. Cell culture was performed for a further $4 \mathrm{~h}$, following which a Fisherbrand ${ }^{\mathrm{TM}}$ accuSkan $^{\mathrm{TM}}$ GO UV/Vis microplate spectrophotometer (Thermo Fisher Scientific, Inc.) was used to measure the optical density values at $450 \mathrm{~nm}$ in order to determine the cell proliferation.

$R T$-qPCR. Total RNA was extracted from cancer tissues, adjacent healthy tissues and serum samples in addition to in vitro cultivated cells using TRIzol reagent (Invitrogen; Thermo Fisher Scientific, Inc.), and miRNAs were isolated using mirVana miRNA Isolation kit (Thermo Fisher Scientific, Inc.). RNA concentrations were measured using NanoDrop ${ }^{\mathrm{TM}}$ 2000c Spectrophotometers (Thermo Fisher Scientific, Inc.). All procedures were performed in strict accordance with the manufacturer's protocol. Total RNA and miRNAs were then reverse transcribed into cDNA using SuperScript III Reverse Transcriptase (Thermo Fisher Scientific, Inc.) and TaqMan MicroRNA Reverse Transcription kit (Thermo Fisher Scientific, Inc.), respectively. Subsequently, qPCR analysis was conducted with Applied Biosystems ${ }^{\mathrm{TM}}$ PowerUp $^{\mathrm{TM}}$ SYBR $^{\mathrm{TM}}$ Green Master Mix (Thermo Fisher Scientific, Inc.) or MystiCq ${ }^{\circledR}$ microRNA ${ }^{\circledR}$ SYBR $^{\circledR}$ Green qPCR ReadyMix ${ }^{\mathrm{TM}}$ (Sigma-Aldrich; Merck KGaA). For the detection of miR-122 expression, the primers used for miR-122 were obtained from Applied Biological Materials, Inc. (cat. no. MPH01045; Richmond, BC, Canada), while the primers of the U6 RNA endogenous control were as follows: 5'-CTCGCTTCGGCA GCACA-3' (forward) and 5'-AACGCTTCACGAATTTGC GT-3' (reverse). For the detection of ROCK2 mRNA expression, the ROCK 2 primers were obtained from Sino Biological, Inc. (cat. no. HP104705; Wayne, PA, USA). Sequences of GAPDH endogenous control were: 5'-CTGACTTCAACA GCGACACC-3' (reverse) and 5'-TAGCCAAATTCGTTG TCATACC-3' (reverse). PCR reactions were performed under following conditions: $50 \mathrm{sec}$ at $95^{\circ} \mathrm{C}$, followed by 40 cycles of $15 \mathrm{sec}$ at $95^{\circ} \mathrm{C}$ and $35 \mathrm{sec}$ at $60^{\circ} \mathrm{C}$. miR-122 and ROCK2 expression levels were normalized to the U6 and GAPDH endogenous control, respectively, using the $2^{-\Delta \Delta \mathrm{Cq}}$ method (12).

Enzyme-linked immunosorbent assay (ELISA). The serum levels of ROCK2 were measured using a human ROCK2 ELISA kit (LS-F22011; LifeSpan BioSciences, Inc., Seattle, WA, USA). All procedures were performed in strict accordance with the manufacturer's protocol.

Western blot analysis. Following total protein extraction from in vitro cultivated cells using radioimmunoprecipitation assay solution (Thermo Fisher Scientific, Inc.), protein concentration was measured by the bicinchoninic acid method, and $20 \mu \mathrm{g}$ protein was then subjected to $10 \%$ SDS-PAGE to separate proteins with different molecular weights. Next, gel transfer was performed, and 5\% skimmed milk was used to block the polyvinylidene difluoride membranes for $1 \mathrm{~h}$ at room temperature. Membranes were then incubated with primary antibodies, including rabbit anti-ROCK2 antibody (1:1,200; ab71598; Abcam, Cambridge, MA, USA) and anti-GAPDH antibody $(1: 1,400 ; \mathrm{ab} 9485 ; \mathrm{Abcam})$ at $4^{\circ} \mathrm{C}$ overnight. The following day, membranes were further incubated with anti-rabbit IgG-HRP secondary antibody (1:1,000; MBS435036; MyBioSource, Inc., 

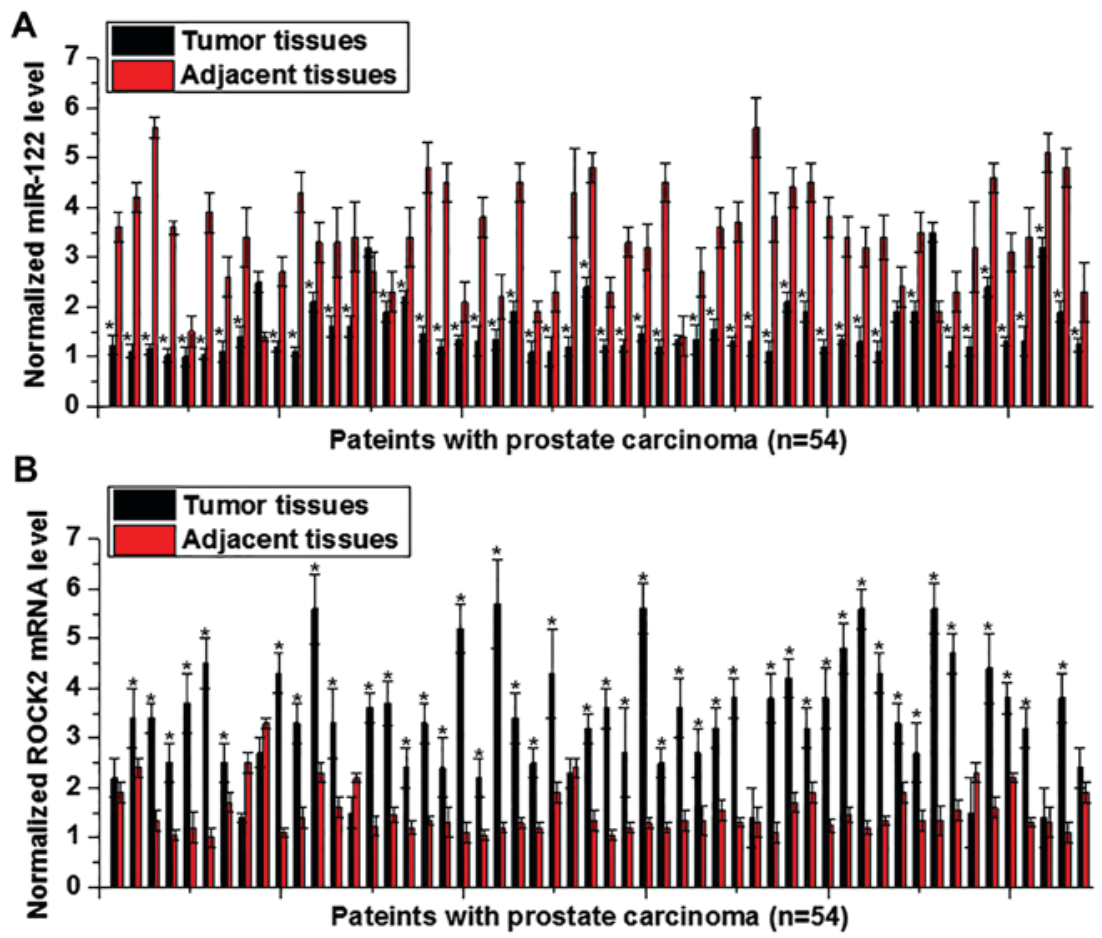

Figure 1. Relative expression levels of (A) miR-122 and (B) ROCK2 mRNA in tumor tissues and adjacent healthy tissues of 54 patients with prostate carcinoma are displayed. " $\mathrm{P}<0.05$ vs. adjacent tissues. miR, microRNA; ROCK2, Rho-associated protein kinase 2.

San Diego, CA, USA) for $2.5 \mathrm{~h}$ at room temperature. Signal development was performed using enhanced chemiluminescence (Sigma-Aldrich; Merck KGaA), and membranes were scanned using MYECL ${ }^{\mathrm{TM}}$ Imager (Thermo Fisher Scientific, Inc.). The expression of ROCK 2 was normalized to that of the GAPDH endogenous control using ImageJ software (version 1.6; National Institutes of Health, Bethesda, MD, USA).

Statistical analysis. All statistical analyses were performed using SPSS software (version 19.0; IBM Corp., Armonk, NY, USA). Protein and mRNA expression levels were expressed as the mean \pm standard deviation. Comparisons between two groups were performed using unpaired t-test, while comparisons among multiple groups were examined by one-way analysis of variance followed by the least significant difference test. Patients were considered to be in the drinking group if they consumed alcohol twice or times more than twice per week, and patients in the smoking group were those who smoked more than 10 cigarettes per day. Correlations between the serum miR-122 level and clinicopathological data of patients were analyzed by $\chi^{2}$ test. Diagnostic value of serum miR-122 in prostate carcinoma was analyzed using receiver operating characteristic (ROC) curve analysis. According to the median serum level of miR-122, the 54 patients with prostate carcinoma were divided into the high $(n=27)$ and low $(n=27)$ expression groups. Survival curves were plotted using the Kaplan-Meier method and compared using the log rank t-test. Differences with $\mathrm{P}<0.05$ were considered as statistically significant.

\section{Results}

Expression levels of miR-122 and ROCK2 mRNA in tumor tissues and adjacent healthy tissues. The expression levels of
miR-122 and ROCK2 mRNA in tumor and adjacent healthy tissues of 54 prostate carcinoma patients were detected by RT-qPCR. As shown in Fig. 1A, a significantly lower expression level of miR-122 in tumor tissues in comparison with that in adjacent tissues was detected in 49 out of 54 patients $(\mathrm{P}<0.05)$, accounting for $90.7 \%$ of cases. By contrast, a significantly higher expression level of ROCK 2 mRNA in tumor tissues as compared with that in adjacent tissues was observed in 46 out of 54 patients $(\mathrm{P}<0.05)$, accounting for $85.2 \%$ of cases. These data suggest that downregulation of miR-122 and upregulation of ROCK2 are likely to be involved in the pathogenesis of prostate carcinoma.

Comparison of serum miR-122 and ROCK2 levels between patients and controls. The serum levels of miR-122 and ROCK 2 in prostate carcinoma patients and healthy controls were measured by RT-qPCR and ELISA, respectively. As shown in Fig. 2, the serum levels of miR-122 were significantly lower (Fig. 2A) and those of ROCK2 were significantly higher (Fig. 2B) in prostate carcinoma patients as compared with those in healthy controls $(\mathrm{P}<0.05)$.

Diagnostic and prognostic value of serum miR-122 level in prostate carcinoma. Data in Fig. 2 indicated that miR-122 was differentially expressed in the serum of prostate carcinoma patients and healthy controls. Therefore, the current study further evaluated the diagnostic value of serum miR-122 in prostate carcinoma using ROC curve analysis. As shown in Fig. 3A, the area under the curve was 0.8954 with a standard error of 0.03186 and a $95 \%$ confidence interval of 0.8330-0.9579. According to the median serum level of miR-122, the 54 patients with prostate carcinoma were divided into the high $(n=27)$ and low $(n=27)$ expression groups. 



Figure 2. Comparison of serum levels of (A) miR-122 and (B) ROCK2 between prostate carcinoma patients and healthy controls. "P<0.05. miR, microRNA; ROCK2, Rho-associated protein kinase 2.


Figure 3. Diagnostic and prognostic value of serum miR-122 level in prostate carcinoma. (A) Receiver operating characteristic curve analysis for the use of serum miR-122 in the diagnosis of prostate carcinoma. (B) Comparison of survival curves of patients in the high and low miR-122 expression groups. miR, microRNA.

Survival curves were plotted using the Kaplan-Meier method and compared using the log rank t-test. As shown in Fig. 3B, the survival time of patient with low serum level of miR-122 was significantly shorter in comparison with that of patients with high serum level of miR-122.

Correlation analysis of serum levels of miR-122 with the clinicopathological data of patients. Correlations between the serum levels of miR-122 and the clinicopathological data of prostate carcinoma patients were analyzed by $\chi^{2}$ test. The results demonstrated that the serum levels of miR-122 were not closely correlated with the patient age, drinking and smoking habits, or distant tumor metastasis. However, the serum levels of miR-122 were significantly correlated with the tumor size.

Effects of miR-122 overexpression on ROCK2 expression. Based on data shown in Table I, it is concluded that miR-122 may be involved in the growth of prostate carcinoma. ROCK2 has been reported to serve a pivotal role in regulating prostate tumor growth (13). Therefore, the effects of miR-122 overexpression on ROCK2 expression were then explored. As compared with the human normal prostate epithelial cell line
HPrEC, the expression levels of miR-122 were significantly lower, and those of ROCK 2 protein were significantly higher in the human prostate carcinoma cell lines 22Rv1 and DU145 (data not shown). As shown in Fig. 4, miR-122 overexpression in 22Rv1 and DU145 prostate carcinoma cells significantly inhibited the expression of ROCK 2 protein, but had no marked effect in normal prostate cells HPrEC.

Effects of miR-122 overexpression and ROCK2 treatment on cell proliferation. As shown in Fig. 5, miR-122 overexpression significantly inhibited the proliferation of human prostate carcinoma 22Rv1 and DU145 cells, whereas it had no marked effect on the normal prostate epithelial cell line HPrEC. In addition, treatment with ROCK2 at a dose of $10 \mathrm{ng} / \mathrm{ml}$ significantly reduced the inhibitory effects of miR-122 overexpression on cell proliferation.

\section{Discussion}

The role of miR-122 has been reported in several malignancies, including hepatocellular carcinoma (9) and oropharyngeal cancer (10); however, its effect in prostate carcinoma remains 
Table I. $\chi^{2}$ analysis of the correlation between the serum levels of miR-122 and the clinicopathological data of patients.

\begin{tabular}{|c|c|c|c|c|c|c|}
\hline \multirow[b]{2}{*}{ Parameter } & \multirow[b]{2}{*}{ Group } & \multirow[b]{2}{*}{ No. of cases } & \multicolumn{2}{|c|}{$\begin{array}{l}\text { miR-122 } \\
\text { expression }\end{array}$} & \multirow[b]{2}{*}{$\chi^{2}$-value } & \multirow[b]{2}{*}{ P-value } \\
\hline & & & High & Low & & \\
\hline \multirow[t]{2}{*}{ Age, years } & $>50$ & 25 & 11 & 14 & \multirow[t]{2}{*}{0.67} & \multirow[t]{2}{*}{0.41} \\
\hline & $<50$ & 29 & 16 & 13 & & \\
\hline \multirow[t]{2}{*}{ Drinking } & Yes & 40 & 18 & 22 & \multirow[t]{2}{*}{1.54} & \multirow[t]{2}{*}{0.21} \\
\hline & No & 14 & 9 & 5 & & \\
\hline \multirow[t]{2}{*}{ Smoking } & Yes & 38 & 17 & 21 & \multirow[t]{2}{*}{0.32} & \multirow[t]{2}{*}{0.57} \\
\hline & No & 16 & 10 & 6 & & \\
\hline \multirow[t]{2}{*}{ Primary tumor diameter, $\mathrm{cm}$} & $>5$ & 22 & 7 & 15 & \multirow[t]{2}{*}{4.91} & \multirow[t]{2}{*}{0.03} \\
\hline & $<5$ & 32 & 20 & 12 & & \\
\hline \multirow[t]{2}{*}{ Tumor distant metastasis } & Yes & 21 & 9 & 12 & \multirow[t]{2}{*}{0.70} & \multirow[t]{2}{*}{0.40} \\
\hline & No & 33 & 18 & 15 & & \\
\hline
\end{tabular}

miR-122, microRNA-122.

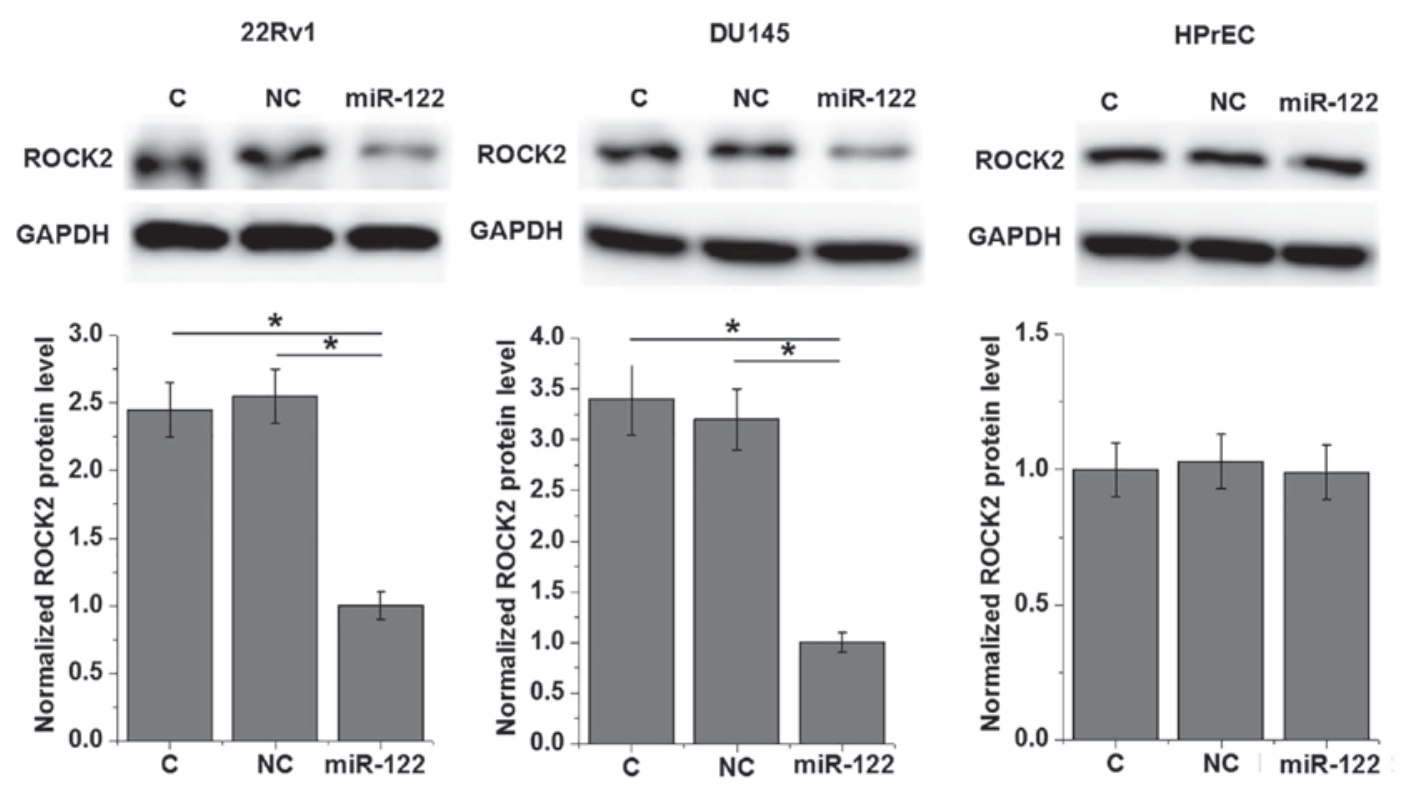

Figure 4. Effects of miR-122 overexpression on ROCK2 protein expression in the prostate carcinoma cell lines 22Rv1 and DU145, and in the human normal prostate epithelial cell line HPrEC. ${ }^{*} \mathrm{P}<0.05$. miR, microRNA; ROCK2, Rho-associated protein kinase 2; C, control; NC, negative control.

unclear. The key finding of the present study was that miR-122 also serves a role as a tumor suppressor gene in prostate carcinoma, and the functionality of miR-122 in this disease may be achieved by inhibiting cell proliferation through the downregulation of ROCK2 expression.

Development of prostate carcinoma is accompanied by changes in the expression pattern of a large set of miRNAs (8), and different miRNAs participate in different stages of prostate carcinoma to promote or inhibit tumor progression (14). It has been reported that miR-96 expression is significantly upregulated in tumor tissues as compared with its expression in adjacent healthy tissues in prostate carcinoma patients, and upregulation of miR-96 is associated with the growth of tumor (15). By contrast, miR-338-3p (16) and miR-195 (17) were downregulated during the development of prostate carcinoma, and overexpression of these two miRNAs exerted tumor suppression effects. Furthermore, significantly downregulated expression of miR-122 in tumor tissues as compared with that in adjacent healthy tissues was observed in patients with hepatocellular carcinoma (9). In the present study, a lower expression level of miR-122 was observed in tumor tissues in comparison with that in paired adjacent tissues in the majority of patients with prostate carcinoma. Therefore, miR-122 may serve as a tumor suppressor miRNA in prostate carcinoma.

miR-122 has been detected in the blood, and circulating miR-122 has been proven to be an effective diagnostic biomarker for hepatocellular carcinoma (18). In the current study, serum levels of miR-122 were also found to be significantly lower in patients with prostate carcinoma as compared with those in healthy controls. The diagnostic potential of this 



Figure 5. Effects of miR-122 overexpression and ROCK2 treatment the proliferation of prostate carcinoma $22 \mathrm{Rv} 1$ and DU145 cells, and normal prostate epithelial HPrEC cells, as investigated by a Cell Counting Kit-8 assay. miR, microRNA; ROCK2, Rho-associated protein kinase 2.

miRNA was evaluated by ROC curve analysis, which revealed that serum miR-122 levels can be used to effectively distinguish patients with prostate carcinoma from normal healthy controls. In addition, low serum levels of miR-122 were proven to be significantly correlated with shorter survival time. These data suggest that serum miR-122 may serve as a potential diagnostic and prognostic biomarker for prostate carcinoma. The current study also demonstrated that the serum levels of miR-122 were not affected by the patient age, or smoking and drinking habits, indicating the high stability of serum miR-122 as a biomarker.

Serum levels of miR-122 were observed to be significantly correlated with tumor size in the present study, but not with distant tumor metastasis, indicating the involvement of miR-122 in the regulation of tumor growth. Subsequent in vitro cell proliferation assay indicated that miR-122 is an inhibitor of prostate carcinoma cell proliferation. ROCK2 signaling has been reported to serve pivotal roles in the tumor development, and upregulated ROCK2 expression was observed in the development of various malignancies, including prostate carcinoma $(11,19)$. The present study results also demonstrated that ROCK2 expression level was higher in tumor tissues when compared with that in adjacent tissues in the majority of patients with prostate carcinoma. The expression of ROCK 2 is known to be regulated by several miRNAs, such as miR-144 (20) and miR-135a (11). In the present study, miR-122 overexpression significantly inhibited the expression of ROCK 2 in prostate carcinoma cells, while ROCK2 treatment significantly reduced the effects of miR-122 overexpression on cell proliferation. Therefore, miR-122 may inhibit the growth of prostate carcinoma by inhibiting cancer cell proliferation through the downregulation of ROCK2 expression. However, ROCK 2 may not be a direct target of miR-122 due to the lack of targeting site on its gene sequence (data not shown). Therefore, disease-associated mediators may exist between ROCK2 and miR-122.

Finally, it is worth noting that miR-122 overexpression only affected the human prostate carcinoma cell lines 22Rv1 and DU145, but had no marked effect on the human normal prostate epithelial cell line HPrEC. Therefore, miR-122 may serve as a potential therapeutic target for prostate carcinoma.

In conclusion, the current study reported that miR-122 expression was downregulated and ROCK 2 expression was upregulated in prostate carcinoma. Thus, low serum level of miR-122 may serve as a marker for prostate carcinoma and an indicator of poor prognosis. Furthermore, miR-122 overexpression inhibited the proliferation of prostate carcinoma cells and the expression of ROCK2, while treatment with ROCK2 attenuated this effect. Therefore, miR-122 may inhibit the proliferation of prostate carcinoma cells possibly by downregulating ROCK2 expression.

\section{Acknowledgements}

Not applicable.

\section{Funding}

The authors would like to thank the National Natural Science Fund (grant no. 30300348) for the financial support provided.

\section{Availability of data and materials}

All data generated or analyzed during the present study are included in this published article.

\section{Authors' contributions}

HL, TH, WJ, YX, XZ and JY were responsible for the conception and design of the study. HL, TH and WJ performed the experiments. YX and $\mathrm{XZ}$ analyzed and interpreted the data. HL and TH drafted the manuscript. HL, TH and JY were responsible for the revision of the manuscript. All authors read and approved the final manuscript.

\section{Ethics approval and consent to participate}

The protocol of the present study was approved by the Ethics Review Committee of Union Hospital, Tongji Medical College, Huazhong University of Science and Technology (Wuhan, China). All participants signed informed consent.

\section{Patient consent for publication}

Not applicable. 


\section{Competing interests}

The authors declare that they have no competing interests.

\section{References}

1. DeSantis CE, Lin CC, Mariotto AB, Siegel RL, Stein KD, Kramer JL, Alteri R, Robbins AS and Jemal A: Cancer treatment and survivorship statistics, 2014. CA Cancer J Clin 64: 252-271, 2014.

2. Nyquist MD and Dehm SM: Interplay between genomic alterations and androgen receptor signaling during prostate cancer development and progression. Horm Cancer 4: 61-69, 2013.

3. Hamid AR, Hoogland AM, Smit F, Jannink S, van Rijt-van de Westerlo C, Jansen CF, van Leenders GJ, Verhaegh GW and Schalken JA: The role of HOXC6 in prostate cancer development. Prostate 75: 1868-1876, 2015.

4. Crawford ED, Stone NN, Evan YY, Koo PJ, Freedland SJ, Slovin SF, Gomella LG, Berger ER, Keane TE, Sieber P, et al: Challenges and recommendations for early identification of metastatic disease in prostate cancer. Urology 83: 664-669, 2014.

5. Ruddon RW: Cancer biology. Oxford University Press, Oxford, 2007.

6. Schröder FH, Hugosson J, Roobol MJ, Tammela TL, Zappa M, Nelen V, Kwiatkowski M, Lujan M, Määttänen L, Lilja H, et al: Screening and prostate cancer mortality: Results of the European randomised study of screening for prostate cancer (ERSPC) at 13 years of follow-up. Lancet 384: 2027-2035, 2014.

7. Chen W, Zheng R, Baade PD, Zhang S, Zeng H, Bray F, Jemal A, Yu XQ and He J: Cancer statistics in China, 2015. CA Cancer J Clin 66: 115-132, 2016

8. Szczyrba J, Löprich E, Wach S, Jung V, Unteregger G, Barth S, Grobholz R, Wieland W, Stöhr R, Hartmann A, et al: The microRNA profile of prostate carcinoma obtained by deep sequencing. Mol Cancer Res 8: 529-538, 2010.

9. Xu J, Zhu X, Wu L, Yang R, Yang Z, Wang Q and Wu F: MicroRNA-122 suppresses cell proliferation and induces cell apoptosis in hepatocellular carcinoma by directly targeting Wnt/ß-catenin pathway. Liver Int 32: 752-760, 2012.

10. Zhang Y, Sturgis EM, Sun Y, Sun C, Wei Q, Huang Z and Li G: A functional variant at miRNA-122 binding site in IL-1 $\alpha$ 3' UTR predicts risk and HPV-positive tumours of oropharyngeal cancer. Eur J Cancer 51: 1415-1423, 2015.
11. Kroiss A, Vincent S, Decaussin-Petrucci M, Meugnier E, Viallet J, Ruffion A, Chalmel F, Samarut J and Allioli N: Androgen-regulated microRNA-135a decreases prostate cancer cell migration and invasion through downregulating ROCK1 and ROCK2. Oncogene 34: 2846-2855, 2015.

12. Livak KJ and Schmittgen TD: Analysis of relative gene expression data using real-time quantitative PCR and the 2(-Delta Delta C(T)) method. Methods 25: 402-408, 2001

13. Zhang C, Zhang S, Zhang Z, He J, Xu Y and Liu S: ROCK has a crucial role in regulating prostate tumor growth through interaction with c-Myc. Oncogene 33: 5582-5591, 2014.

14. Hart M, Nolte E, Wach S, Szczyrba J, Taubert H, Rau TT, Hartmann A, Grässer FA and Wullich B: Comparative microRNA profiling of prostate carcinomas with increasing tumor stage by deep sequencing. Mol Cancer Res 12: 250-263, 2014.

15. Xu L, Zhong J, Guo B, Zhu Q, Liang H, Wen N, Yun W and Zhang L: miR-96 promotes the growth of prostate carcinoma cells by suppressing MTSS1. Tumour Biol 37: 12023-12032, 2016.

16. Bakkar A, Alshalalfa M, Petersen LF, Abou-Ouf H, Al-Mami A, Hegazy SA, Feng F, Alhajj R, Bijian K, Alaoui-Jamali MA and Bismar TA: microRNA 338-3p exhibits tumor suppressor role and its down-regulation is associated with adverse clinical outcome in prostate cancer patients. Mol Biol Rep 43: 229-240, 2016.

17. Guo J, Wang M and Liu X: MicroRNA-195 suppresses tumor cell proliferation and metastasis by directly targeting BCOX1 in prostate carcinoma. J Exp Clin Cancer Res 34: 91, 2015.

18. El-Abd NE, Fawzy NA, El-Sheikh SM and Soliman ME: Circulating miRNA-122, miRNA-199a, and miRNA-16 as biomarkers for early detection of hepatocellular carcinoma in egyptian patients with chronic hepatitis $\mathrm{C}$ virus infection. Mol Diagn Ther 19: 213-220, 2015.

19. Li M, Ke J, Wang Q, Qian H, Yang L, Zhang X, Xiao J, Ding H, Shan X, Liu Q, et al: Upregulation of ROCK2 in gastric cancer cell promotes tumor cell proliferation, metastasis and invasion. Clin Exp Med 17: 519-529, 2017.

20. Wang W, Zhou X and Wei M: MicroRNA-144 suppresses osteosarcoma growth and metastasis by targeting ROCK1 and ROCK2. Oncotarget 6: 10297-10308, 2015. 